\title{
Serpin peptidase inhibitor clade A member 1 is a biomarker of poor prognosis in gastric cancer
}

\author{
C H Kwon ${ }^{1,4}$, H J Park ${ }^{1,4}$, J R Lee ${ }^{1}, \mathrm{H} \mathrm{K} \mathrm{Kim}{ }^{1}, \mathrm{~T}$ Y Jeon ${ }^{2}, \mathrm{H}-\mathrm{J} \mathrm{Jo}{ }^{2}$, D H Kim ${ }^{2}, \mathrm{G} \mathrm{H} \mathrm{Kim}{ }^{3}$ and D Y Park ${ }^{*, 1}$
}

${ }^{1}$ Department of Pathology, Pusan National University Hospital and Pusan National University School of Medicine, and BioMedical Research Institute, Pusan National University Hospital, 1-10 Ami-Dong, Seo-Gu, Busan 602-739, Korea; ${ }^{2}$ Department of Surgery, Pusan National University Hospital and Pusan National University School of Medicine, and BioMedical Research Institute, Pusan National University Hospital, 1-10 Ami-Dong, Seo-Gu, Busan 602-739, Korea and ${ }^{3}$ Department of Internal Medicine, Pusan National University Hospital and Pusan National University School of Medicine, and BioMedical Research Institute, Pusan National University Hospital, 1-10 Ami-Dong, Seo-Gu, Busan 602-739, Korea

Background: In a previous study, we reported that serpin peptidase inhibitor clade A member 1 (serpinA1) is upregulated in Snail-overexpressing gastric cancer. Although serpinA1 has been studied in several types of cancer, little is known about its roles and mechanisms of action. In this study, we examined the role of serpinA1 in the migration and invasion of gastric cancers and determined its underlying mechanism.

Methods: Expression levels were assessed by western blot analyses and real-time PCR. Snail binding to serpinA1 promoter was analysed by chromatin immunoprecipitation (ChIP) assays. The roles of serpinA1 were studied using cell invasion and migration assays. In addition, the clinicopathologic and prognostic significance of serpinA1 expression were validated in 400 gastric cancer patients using immunohistochemical analysis.

Results: Overexpression of Snail resulted in upregulation of serpinA1 in gastric cancer cell lines, AGS and MKN45, whereas knockdown of Snail inhibited serpinA1 expression. Chromatin immunoprecipitation analysis showed that overexpression of Snail increased Snail recruitment to the serpinA1 promoter. Overexpression of serpinA1 increased the migration and invasion of gastric cancer cells, whereas knockdown of serpinA1 decreased invasion and migration. Moreover, serpinA1 increased mRNA levels and release of metalloproteinase-8 in gastric cancer cells. Serpin peptidase inhibitor clade A member 1 was observed in the cytoplasm of tumour cells and the stroma by immunohistochemistry. Enhanced serpinA1 expression was significantly associated with increased tumour size, advanced T stage, perineural invasion, lymphovascular invasion, lymph node metastases, and shorter overall survival.

Conclusions: Serpin peptidase inhibitor clade A member 1 induces the invasion and migration of gastric cancer cells and its expression is associated with the progression of gastric cancer. These results may provide a potential target to prevent invasion and metastasis in gastric cancer.

Gastric cancer has one of the highest mortality rates worldwide and ranks second globally in cancer-related disease mortality (Parkin et al, 2005). The majority of gastric cancers are classified as adenocarcinomas, which are characterised by a variety of anatomic and histologic features, as well as various clinical progressions in the patient. Preoperative staging techniques for gastric cancer,

${ }^{*}$ Correspondence: Professor DY Park; E-mail: pdy220@pusan.ac.kr
${ }^{4}$ These authors contributed equally to this work

Revised 24 July 2014; accepted 13 August 2014; published online 11 September 2014

(c) 2014 Cancer Research UK. All rights reserved 0007-0920/14 
including laparoscopy and noninvasive imaging systems, have relatively low sensitivity for discriminating patients (Roukos and Kappas, 2005). Moreover, the best available gastric cancer treatment remains surgical resection and patients are treated with similar agents following curative resection (Kovoor and Hwang, 2009), resulting in combined sequelae of extensive gastric resection and side effects of antitumour chemotherapy. Therefore, the development of a gastric cancer biomarker that would enable accurate determination of onset and progression staging, as well as accurate prognosis, is essential. To identify novel biomarkers, we have analysed gene expression profiling data from gastric cancer patients. In a previous study of gastric cancer, we found that Snail expression was associated with prognosis and that serpin peptidase inhibitor clade A member 1 (serpinA1) expression was increased in Snail-overexpressing tissues of gastric cancer patients (Shin et al, 2012).

Serine protease inhibitors (serpins) belong to the protease inhibitor family and are classified as either 'clade A', comprising the extracellular molecules, or 'clade B', the intracellular serpins (Siverman et al, 2001; Law et al, 2006). Serpin peptidase inhibitor clade A member 1 (serpinA1) is also known as 1-proteinase inhibitor or 1-antitrypsin (AAT). Serpin peptidase inhibitor clade A member 1 is primarily synthesised in the liver and is also produced in certain cells, such as gastric cancer, colon cancer, and lung cancer cells (Karashima et al, 1990; Higashiyama et al, 1992; Allgayer et al, 1998). Tumour cells synthesise and release not only a native form of serpinA1 but also a variety of cleaved and/or degraded forms of serpinA1 (Zelvyte et al, 2002). These protease inhibitors have major roles in physiologic and pathologic processes such as angiogenesis, intravascular fibrinolysis, wound healing, and tumour invasion and metastasis (Janciauskiene, 2001; Brantly, 2002). The expression of serpinAl has been reported to be correlated with a poor prognosis in terms of metastasis in lung cancer, colon cancer, and squamous cell cancer patients (Karashima et al, 1990; Higashiyama et al, 1992; Farshchian et al, 2011). Furthermore, with respect to gastric cancer, serpinA1 expression was also found to increase and to be correlated with a poor prognosis in well-differentiated adenocarcinoma (Tahara et al, 1984). However, the detailed mechanisms of serpinAl in the progression and metastasis of gastric cancer remain unknown, and no correlation between the expression of serpinA1 with Snail has been reported. Moreover, although the effect of serpinA1 has been studied in several cancers, no existing analyses have clarified the correlation between these relevant clinical features, especially in gastric cancer.

Therefore, the aim of this study was to elucidate the functions and underlying mechanism of serpinAl in gastric cancer cells, particularly the relationship between Snail and serpinA1. Moreover, the viability of serpinA1 as a biomarker was investigated via confirmation of serpinA 1 expression in tissues from gastric cancer patients and investigation of the association between serpinA1 expression and the clinicopathologic characteristics of gastric cancer patients.

\section{MATERIALS AND METHODS}

Cell lines and transfection. The human gastric cancer cell lines MKN45 and AGS were obtained from the Korean Cell Line Bank (Seoul, South Korea) and were authenticated by DNA profiling. The DNA printing of cell lines were tested using STR Kit (Applied Biosystems, Foster City, CA, USA) and MKN45 were initially characterised (Kitano and Kitamura, 1993). These cells were cultured in RPMI1640 medium with 10\% fetal bovine serum (FBS; Gibco, Grand Island, NY, USA), $100 \mathrm{U} \mathrm{ml}^{-1}$ penicillin, and $100 \mu \mathrm{g} \mathrm{ml}^{-1}$ streptomycin (Sigma-Aldrich, St Louis, MO, USA). All cells were maintained at $37^{\circ} \mathrm{C}$ in $5 \% \mathrm{CO}_{2}$.
For overexpression of genes, cells were grown to 60-70\% confluence and transfected with the pcDNA Snail-Myc or pcDNA SerpinA1-Myc vectors (Origene, Rockville, MD, USA), or with the pcDNA-3.1 vector as a control, with Lipofectamine 2000 (Invitrogen, Carlsbad, CA, USA) according to the manufacturer's protocol. Cells were maintained in complete medium for $24 \mathrm{~h}$ before the assays were performed.

For knockdown of genes, cells were transfected with Snail or serpinA1 smartpool short interfering RNA (siRNA) or with non-targeting siRNA as a control (Dharmacon, Pittsburgh, PA, USA), using Lipofectamine RNAiMAX reagent (Invitrogen) according to the manufacturer's instructions. The siRNA sequences were as follows: Snail siRNA, 5'-GCGAGCUGCAGGACUCUAA-3', 5'-AAUCGGAAGCCUAACUACA-3', 5'-GUGACUAACUACUGC AAUAA-3' ${ }^{\prime}$, ${ }^{\prime}$-GAGUAAUGGCUGUCACUUG-3'; serpinA1 siRNA, $5^{\prime}$-GAACUCACCCACGAUAUCA-3', $5^{\prime}$-GAUGAAGCGUUUAGG CAUG-3', 5'-CCUAUGAUCUGAAGAGCGU-3', 5'-CCAAGAAA CAGAUCAACGA- $3^{\prime}$.

Real-time reverse transcription-PCR analysis. Total RNA was extracted from gastric cancer cells, using the TRIzol reagent (Invitrogen), following the manufacturer's instructions. RNA was reverse transcribed with SuperScript II (Invitrogen) and cDNA was amplified with each primer and visualised with SYBR Green (Applied Biosystems), using the fluorescence reader Corbett Rotor-Gene 6000 (Qiagen Inc., Valencia, CA, USA). The primers used were as follows: glyceraldehyde 3-phosphate dehydrogenase (GAPDH), 5'-TCCATGACAACTTTGGTATCG-3', 5'-TGTAGC CAAATTCGTTGTCA-3'; serpinA1, $5^{\prime}$-GGAACAGGCTCAGG ACTATCTC- $3^{\prime}, 5^{\prime}$-CAACATCTGGCACTCCACA-3'; and metalloproteinase-8 (MMP8), 5'-TTGCTGCTCTACCTCCACCA-3', $5^{\prime}$-GCACACAGGATGGCTTGAA- $3^{\prime}$. The following thermal cycler programme was used: denaturation for $30 \mathrm{~s}$ at $95^{\circ} \mathrm{C}$; annealing for $30 \mathrm{~s}$ at $52^{\circ} \mathrm{C}$, depending on the primers used; and extension for $30 \mathrm{~s}$ at $72{ }^{\circ} \mathrm{C}$. The number of PCR cycles was determined for each gene and ranged from 25 to 35 . Data were normalised to GAPDH, and mRNA abundance was calculated using the $2^{-\Delta \Delta C T}$ method. The PCR products were confirmed by mobility on gel electrophoresis.

Chromatin immunoprecipitation assays. Chromatin immunoprecipitation assays were performed using the EZ-ChIP Kit (Merck Millipore, Darmstadt, Germany). Cells were crosslinked with 2\% formaldehyde for $10 \mathrm{~min}$ at $37^{\circ} \mathrm{C}$ and washed in ice-cold PBS. Unreacted formaldehyde was quenched with $200 \mathrm{~mm}$ glycine, then cells were washed with PBS, and finally resuspended in SDS lysis buffer containing protease inhibitors. Samples were sheared by sonication on ice, diluted in dilution buffer with inhibitors, and precleared with agarose $G$ beads. A portion of the supernatant was stored as input, and the remaining supernatant was divided for immunoprecipitation and incubated with anti-Snail (R\&D System, Minneapolis, MN, USA) or IgG as a negative control overnight at $4{ }^{\circ} \mathrm{C}$ with agitation. Immune complexes were captured using protein A-sepharose, then washed sequentially in low-salt buffer, high-salt buffer, two times in $\mathrm{LiCl}$ buffer, and finally two times in TE buffer. Protein was eluted from the beads in fresh elution buffer, crosslinking was reversed overnight at $65^{\circ} \mathrm{C}$ in the presence of $\mathrm{NaCl}$, and then samples were ethanol precipitated. Following centrifugation, pellets were resuspended in TE buffer and incubated sequentially with $50 \mu \mathrm{g} \mathrm{ml}^{-1}$ RNase A (30 min) and $100 \mu \mathrm{g} \mathrm{ml}^{-1}$ proteinase $\mathrm{K}(1 \mathrm{~h})$. DNA was purified by washing with elution buffer and centrifugation and then finally analysed by real-time PCR. The primer sequence was $5^{\prime}$-AAAGAGCAG GACCCCAAAT- $3^{\prime}$ and 5'-TCCACCCGAAGTCTACTTCC- ${ }^{\prime}$.

Cell migration and invasion assays. Gastric cancer cells were harvested with $0.05 \%$ trypsin containing $0.02 \%$ EDTA (Sigma-Aldrich) and suspended in RPMI medium. For the 
migration assay, membrane filters $(8-\mu \mathrm{m}$ pore size) in disposable 96-well chemotaxis chambers (Neuro Probe, Gaithersburg, MD, USA) were precoated with $5 \mathrm{mg} \mathrm{ml}^{-1}$ fibronectin for $4 \mathrm{~h}$ at room temperature. Cells $\left(3 \times 10^{3}\right.$ cells per well $)$ were loaded into the upper chambers, and 1\% FBS was loaded into the lower chamber. After $24 \mathrm{~h}$ of incubation, non-migrating cells were removed from the upper chamber with a cotton swab, and the cells on the lower surface of the insert were stained with Hoechst33342 (Sigma-Aldrich). Migrated cells were counted under a fluorescence microscope at $\times 10$ magnification.

For the invasion assay, $3 \times 10^{4}$ cells per well were seeded in the upper chamber, which was coated with $30 \mu \mathrm{l}$ of Matrigel $\left(1 \mathrm{mg} \mathrm{ml}^{-1}\right.$ in cold medium; BD Transduction Laboratories, Franklin Lakes, NJ, USA). Serum-free medium containing $1 \%$ FBS or control vehicle was added to the lower chamber. After $24 \mathrm{~h}$ of incubation, non-invading cells were removed from the upper chamber with a cotton swab, and cells on the lower surface of the insert were stained with Hoechst33342 (Sigma-Aldrich). Invasive cells were counted under a fluorescence microscope at $\times 10$ magnification.

Western blot analysis. Cells were harvested and disrupted in lysis buffer (1\% Triton X-100, 1 mm EGTA, 1 mm EDTA, $10 \mathrm{~mm}$

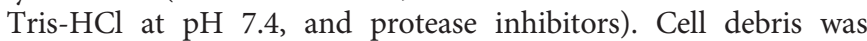
removed via centrifugation at $10000 \mathrm{~g}$ for $10 \mathrm{~min}$ at $4{ }^{\circ} \mathrm{C}$. The resulting supernatants were resolved using SDS-PAGE and transferred onto nitrocellulose membranes. The membranes were blocked with $5 \%$ non-fat dried milk at room temperature for $30 \mathrm{~min}$ and incubated with anti-Snail (AF3639; R\&D Systems), anti-serpinA1 (HPA000927; Sigma-Aldrich), and anti-GAPDH. The membranes were then washed and incubated with horseradish peroxidase-conjugated secondary antibody. Signals were visualised using enhanced chemiluminescence (Amersham, Buckinghamshire, UK).

ELISA. Cells were plated in 6-well tissue culture plates and the supernatants were collected $24 \mathrm{~h}$ after plating. The concentrations of MMP8 were determined using Quantikine ELISA Kits, according to the manufacturer's instructions (R\&D Systems).

Immunohistochemistry and analysis of clinicopathologic and prognostic significance. Standard formalin-fixed and paraffin-embedded sections were obtained from the Department of Pathology and the National Biobank of Korea, Pusan National University Hospital (PNUH). All samples from the National Biobank of Korea were obtained with informed consent under institutional review board-approved protocols. Slides were baked at $60{ }^{\circ} \mathrm{C}$ for $30 \mathrm{~min}$, deparaffinised with xylene, and rehydrated. The sections were submerged in citrate antigen retrieval buffer, heated in a microwave for antigen retrieval, treated with $3 \%$ hydrogen peroxide in methanol to quench endogenous peroxidase activity, and incubated with $1 \%$ bovine serum albumin to block nonspecific binding. The sections were incubated with serpinAl antibody (1:500 dilution; HPA000927; Sigma-Aldrich) overnight at $4{ }^{\circ} \mathrm{C}$. Normal rabbit serum was used as a negative control. After washing, tissue sections were treated with secondary antibody, counterstained with haematoxylin, dehydrated, and mounted. Serpin peptidase inhibitor clade A member 1 immunostaining was scored semiquantitatively as follows: negative $(-)$, weak $(+)$, moderate $(++)$, and strong $(+++)$.

We studied a cohort of 400 gastric cancer patients who underwent gastrectomy with lymph node dissection at PNUH between 2005 and 2007 - the same cohort used in an earlier study (Kim et al, 2013). None of the patients received preoperative radiotherapy or chemotherapy. We assessed the following clinicopathologic factors according to the Korean Standardized Pathology Report for Gastric Cancer and the American Joint Committee on Cancer Staging Manual, 7th edition: site, gross type, tumour size, depth of invasion, histologic classification (i.e., intestinal or diffuse), and lymphovascular invasion (Kim et al, 2005; Edge et al, 2009). The average follow-up period was 68.33 months. Cases lost to follow-up or death from any other causes were defined as censored data for the analysis of survival rates.

Clinicopathologic features were analysed for differences in serpinA1 expression using the Student's $t$-test, the $\chi^{2}$ test, or Fisher's exact test. The relationships between expression of tumour cells and the stroma were assessed with a Spearman's rank correlation coefficient. For statistical analysis of tumour cells, semiquantitative immunostaining results were regrouped so that negative $(-)$ and weak $(+)$ staining results were considered as one group, and moderate $(++)$ and strong $(+++)$ semiquantitative results were considered as another group. Cumulative survival plots were obtained using the Kaplan-Meier method, and significance was compared using the log-rank test. Statistical significance was set at $P<0.05$. Statistical calculations were performed using SSPS version 10.0 for Windows (SPSS Inc., Chicago, IL, USA).

\section{RESULTS}

SerpinA1 is regulated by Snail. We previously conducted a microarray analysis of 48 frozen tissue samples from gastric cancer patients. In this previous study, serpinA1 expression was elevated in Snail-overexpressing gastric cancer patients (Shin et al, 2012). To investigate the dependence of Snail on serpinAl expression, gastric cancer cell lines, MKN45 and AGS, were transfected with pcDNA-Snail, or pcDNA-3.1 as a control. Expression of Snail and serpinA1 was detected by western blot analysis. The results showed that protein levels of serpinA1 were increased in Snail-overexpressing cells compared with control cells (Figure 1A). Conversely, RNA interference-mediated knockdown of Snail led to reduced serpinA1 expression (Figure 1B). Next, to examine whether serpinA1 had an inverse effect on Snail expression, cells were transfected with pcDNA-serpinA1 to induce overexpression, or serpinA1-targeting siRNA to induce knockdown. Snail expression was not altered by serpinA1 (Figure $1 \mathrm{C}$ and D). These data suggest that serpinA1 is a downstream mediator of Snail and is concordant with the previous microarray results, which indicated that serpinA1 expression was increased in Snail-overexpressing gastric cancer patients.

Snail is a zinc-finger transcription factor that contributes to the acquisition of invasive properties in tumours (Miyoshi et al, 2005; Olmeda et al, 2007). Thus, we tested whether Snail binds to the proximal serpinA1 promoter to study the mechanism that controls serpinA1. We performed a ChIP assay with Snail antibody in MKN45 and AGS cells transfected with pcDNA-Snail, or pcDNA3.1 as a control, and performed real-time PCR using serpinA1 promoter primers. We observed that Snail antibody precipitated the serpinA1 promoter more strongly in Snail-overexpressing cells than control cells (Figure $1 \mathrm{E}$ and F). Mouse IgG was used as a negative control. These results indicate that overexpression of Snail may lead to an increase in serpinAl expression, in part through binding of Snail to the serpinA1 promoter, in gastric cancer cells.

SerpinA1 promotes invasion and migration. Tumour cells have invasive and migratory capabilities, which promote the dissemination of tumour cells to distant organs (Thiery et al, 2009). Thus, to investigate the role of serpinA1 in migration and invasion of gastric cancer cells, MKN-45 and AGS cells were transfected with pcDNA-serpinA1 or control vector pcDNA3.1, and then their migration and invasion abilities were measured using transwell assays. Western blot analysis showed that expression of serpinA1 was increased in both cell lines after transfection with pcDNA-serpinA1 (Figure 2A). As shown in Figures 2B-E, 

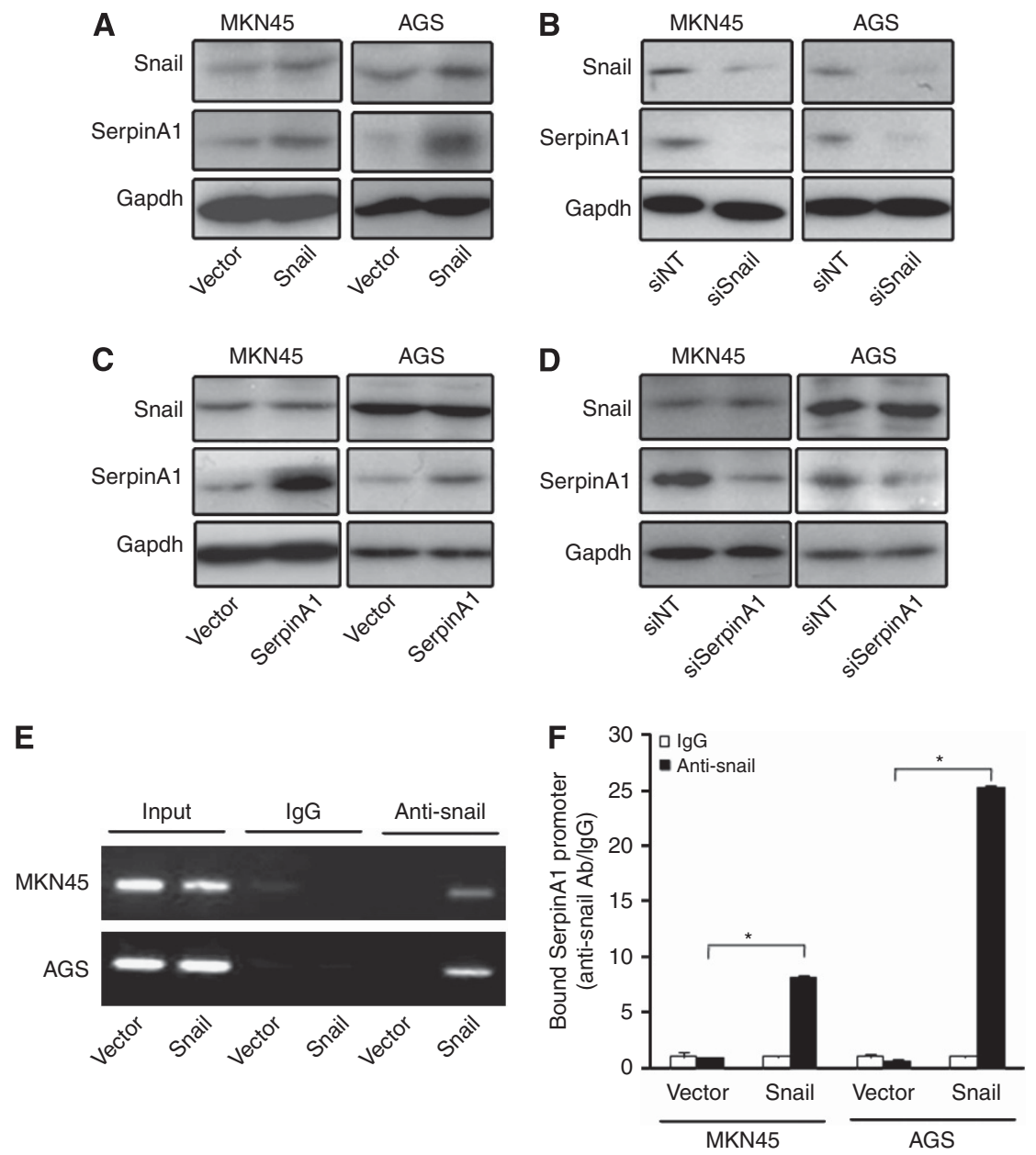

Figure 1. SerpinA1 is regulated by Snail. (A and B) MKN45 and AGS cells were transfected with pcDNA-Snail (Snail), control vector pcDNA (vector), Snail siRNA (siSnail), or non-targeting siRNA (siNT) and protein levels of Snail and serpinA1 were evaluated by western blot analysis. (C and D) MKN45 and AGS cells were transfected with pcDNA-serpinA1 (serpinA1), control vector pcDNA (vector), serpinA1 siRNA (siserpinA1), or non-targeting siRNA (siNT) followed by western blot analysis for Snail and SerpinA1 expression. (E) MKN45 and AGS cells were transfected with pcDNA-Snail (Snail) or control vector pcDNA (vector) to perform the ChIP assay. Presence of the serpinA1 promoter ( $-516 /-4)$ was verified inimmunoprecipitates with either mouse IgG or anti-Snail antibody and assay inputs were analysed using real-time PCR. The samples were loaded on agarose gels. (F) Data show promoter enrichment in the anti-Snail immunoprecipitate relative to lgG.

migration and invasion were increased by overexpression of serpinA1 in gastric cancer cells.

In addition, we performed knockdown assays to verify that serpinA1 promotes the invasion and migration of gastric cancer cells. Cells were transfected with serpinA1-targeting siRNA (siSerpinA1) or non-targeting siRNA (siNT) as a control. Expression levels of serpinA1 were significantly lower in MKN45 and AGS cells transfected with siSerpinA1 than in cells transfected with siNT (Figure 2F). Next, we evaluated migration and invasion responses. Migration and invasion were attenuated by serpinA1 knockdown in gastric cancer cells (Figure 2G-J). These results suggest that serpinA1 has an essential role in the migration and invasion of gastric cancer cells.

SerpinA1 upregulates MMP8. To further investigate the mechanisms by which serpinA1 promotes invasion and migration, we evaluated levels of MMPs, which are associated with tumour invasiveness. Reverse transcription-PCR analysis showed that overexpression of serpinAl significantly increased the mRNA levels of MMP8 in MKN45 and AGS cells compared with control cells (Figure 3A). In contrast, abrogation of serpinA1 by transfection with siSerpinA1 reduced levels of MMP8, compared with cells expressing siNT (Figure 3D). Similar results were obtained for real-time PCR (Figure $3 \mathrm{~B}$ and $\mathrm{E}$ ) and for ELISA of conditioned medium obtained from MKN45 and AGS cells (Figure 3C and F).

SerpinA1 expression is associated with progression and poor outcomes in gastric cancer. SerpinA1 expression was observed in the cytoplasm of tumour cells and adjacent stromal tissue (Figure 4). In tumour cells, negative $(-)$ or weak $(+)$ serpinA1 staining was observed in 249 of 400 cases (62.25\%) (Figure $4 \mathrm{~A}$ and $\mathrm{B})$; moderate $(++)$ or strong $(+++)$ cytoplasmic staining was noted in 151 of 400 cases (37.75\%) (Figures $4 \mathrm{C}$ and D). For stromal serpinA1 expression, most gastric cancer tissue showed negative $(-)(19.8 \%)$ (Figure $4 \mathrm{~B})$ or weak $(+)(71.8 \%)$ (Figure $4 \mathrm{C}$ and D) staining and a small number of samples (8.5\%) showed moderate staining $(++)$ intensity (Figure $4 \mathrm{~A})$. Serpin peptidase inhibitor clade A member 1 expression in tumour cells showed a strong correlation with that in the stroma $(P<0.0001, r=0.445$; data not shown).

Immunohistochemical staining for serpinA1 was statistically analysed to determine its relationship with clinicopathologic features (Tables 1 and 2). Serpin peptidase inhibitor clade A member 1 overexpression in tumour cells was strongly correlated with mean patient age $(P=0.003)$ and mean tumour size 

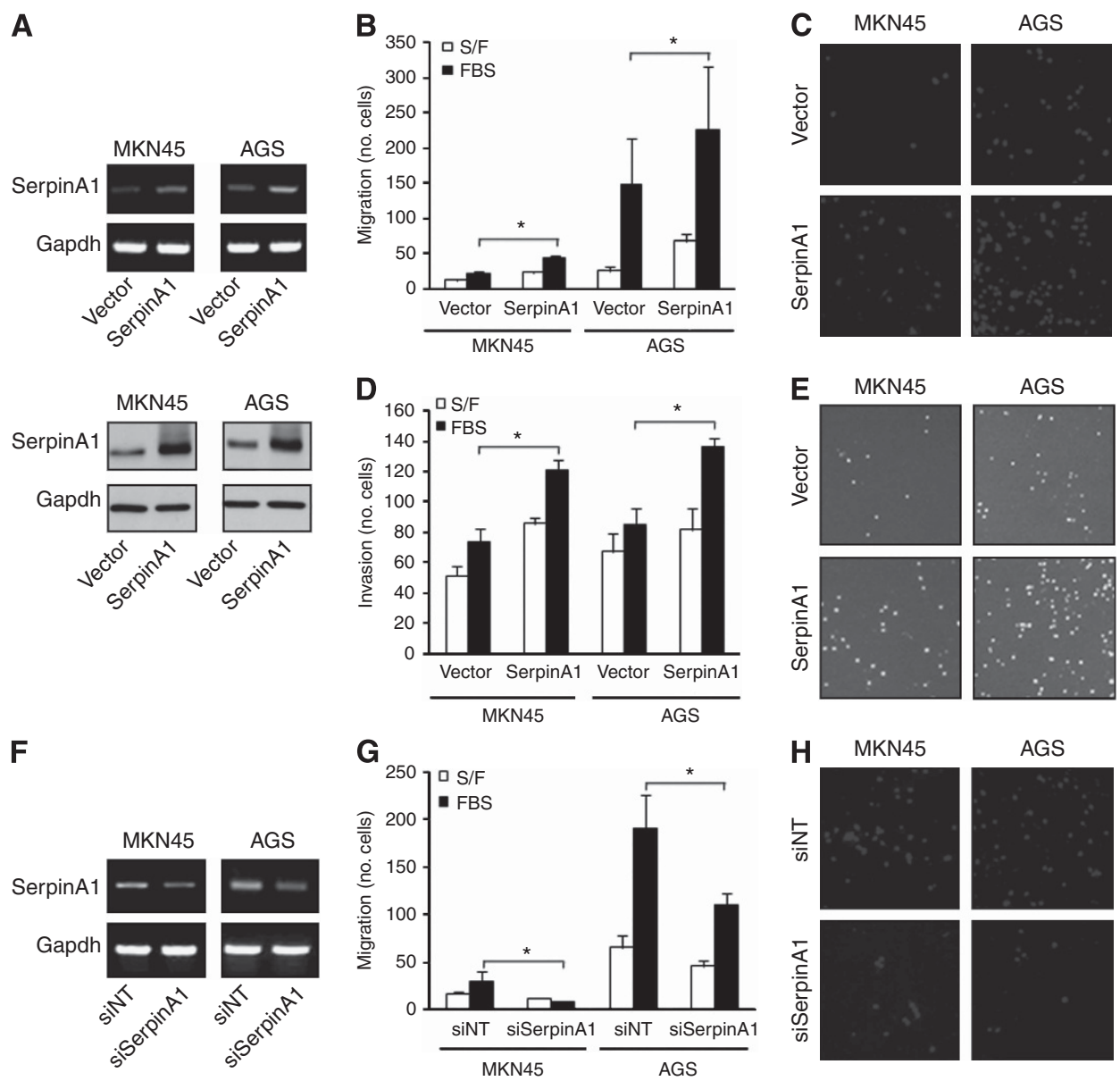

\section{AGS}
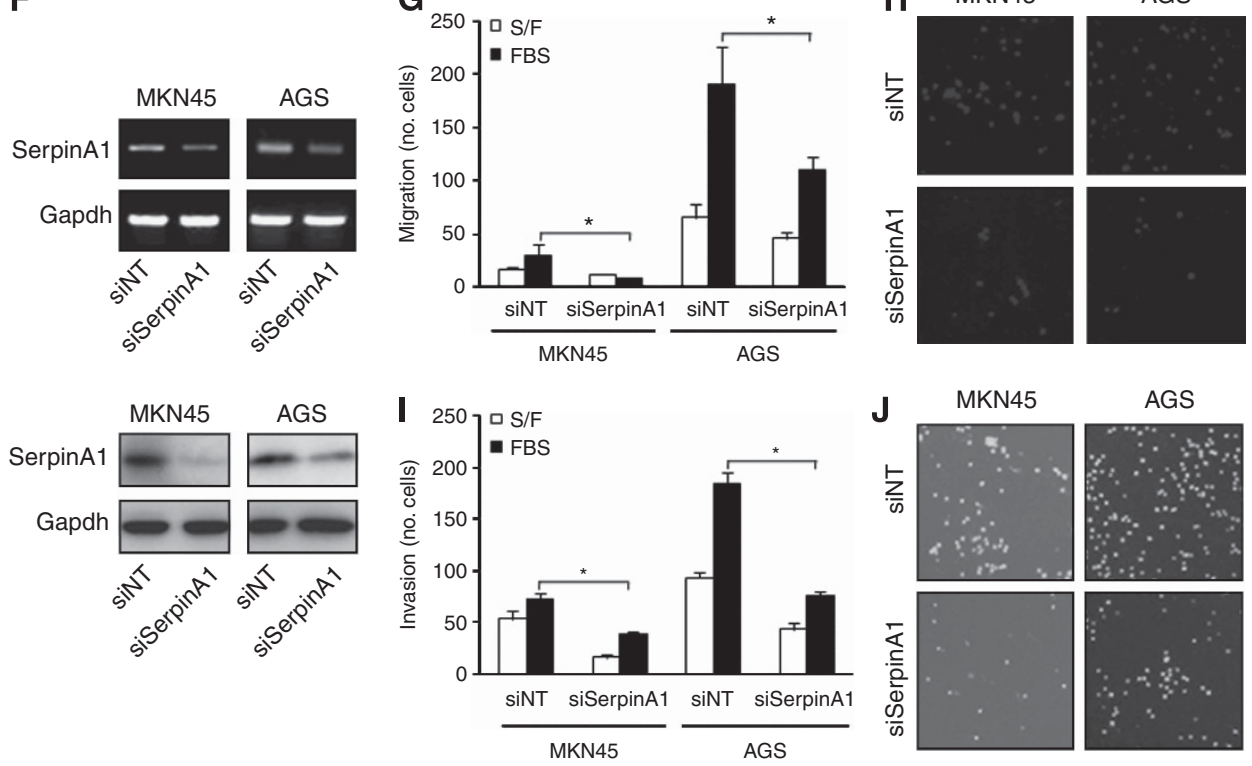

AGS

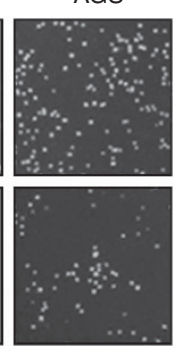

Figure 2. Effect of serpinA1 on gastric cancer invasion and migration. (A) MKN45 and AGS cells were transfected with pcDNA-serpinA1 (serpinA1) or control vector pcDNA (vector) for migration and invasion assays. mRNA and protein levels of serpinA1 were evaluated using RT-PCR and western blot analysis. (B) Transfected cells were allowed to migrate to chemotaxis chambers for $24 \mathrm{~h}$ in the presence or absence of $1 \% \mathrm{FBS}$. The migrated cells were fixed, stained, photographed, and counted. Data show the average of four independent experiments. ${ }^{\star} P<0.05$. (C) The representative data are shown for cells that migrated in the presence of $1 \%$ FBS. (D) Cells that had spread through the Matrigel and into the lower surface of the filter were fixed, stained, photographed, and counted. Data show the average of four independent experiments. ${ }^{*} P<0.05$. (E) The representative data are shown for cells that invaded in the presence of $1 \%$ FBS. (F) MKN45 and AGS cells were transfected with serpinA1 siRNA (siSerpinA1) or non-targeting siRNA (siNT) for migration and invasion assays. mRNA and protein levels of serpinA1 were evaluated using RT-PCR and western blotting. Migration ( $\mathbf{G}$ and $\mathbf{H})$ and invasive ability $(\mathbf{I}$ and $\mathbf{J}$ ) were evaluated using chemotaxis chambers and Matrigel-coated transwells, respectively. Data represent the average of four independent experiments. ${ }^{*} P<0.05$. The representative data are shown for cells that migrated or invaded in the presence of $1 \%$ FBS.

$(P=0.003)$. However, serpinA1 expression was not significantly correlated with patient's sex and the tumour location. With respect to gross type, serpinAl expression was higher in excavated-type (79 cases $(50.0 \%), P<0.0001)$ than in elevated-type (47 cases $(51.6 \%))$ or flat/depressed-type tumours (25 cases (16.6\%)). With respect to histologic type, significantly increased serpinA 1 expression $(P=0.002)$ was observed in mixed-type $(1(25.0 \%))$ and intestinal-type tumours $(102(44.5 \%))$ relative to diffuse-type tumours $(48(28.7 \%))$.With respect to invasion depth, serpinA1 overexpression was significantly higher $(P<0.0001)$ in T2 (29 $(47.5 \%))$, T3 (36 (56.2\%)), and T4tumours $(42(48.8 \%))$ than in T1 tumours $(44(23.3 \%))$. The frequency of serpinA1 overexpression was higher in the perineural invasion-positive group $(76(48.4 \%))$ than in the perineural invasion-negative group (75 (30.9\%); $P<0.0001)$. Moreover, serpinA1 was more frequently overexpressed in the lymphovascular emboli-positive group $(79(52.7 \%))$ than in the lymphovascular emboli-negative group (72 $(28.8 \%) ; P<0.0001)$. With respect to lymph node metastasis, 

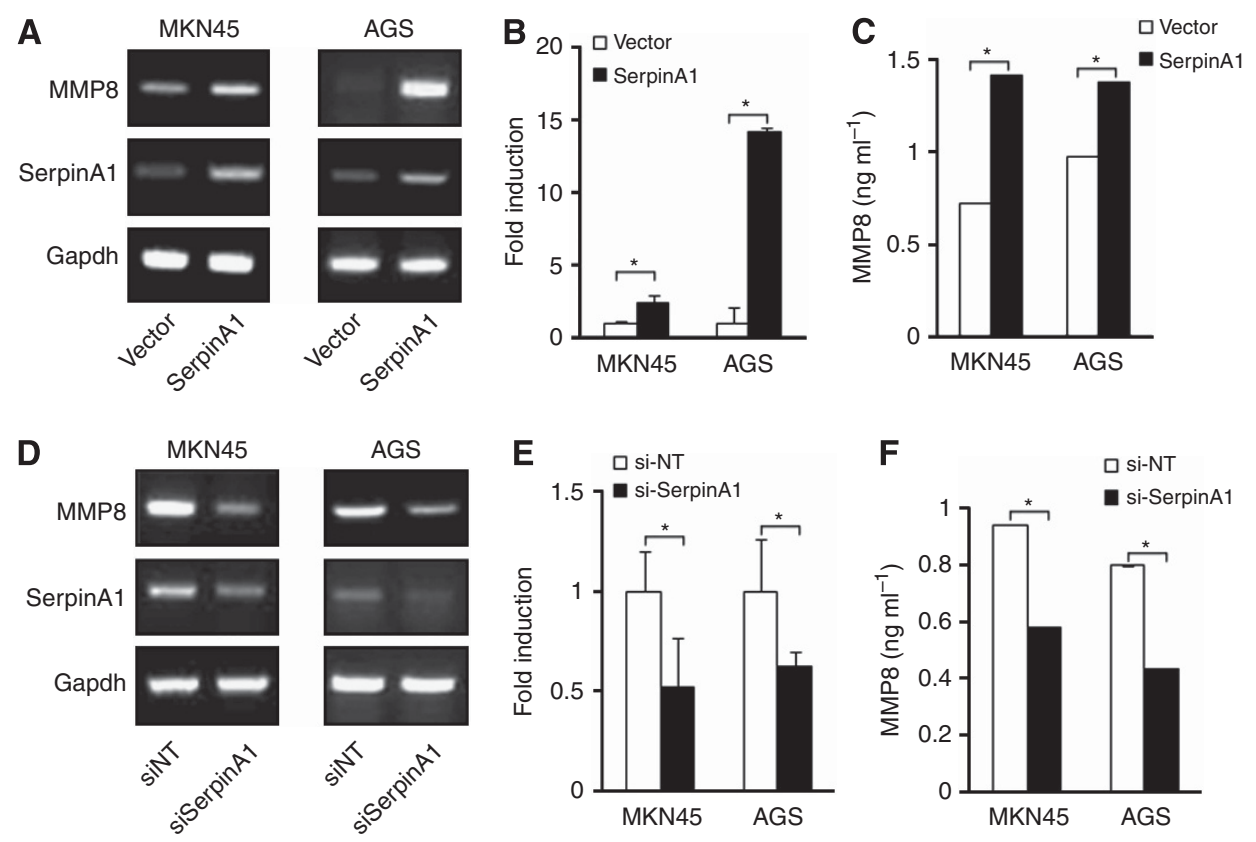

Figure 3. Effect of serpinA1 on MMP8 expression. MKN45 and AGS cells were transfected with pcDNA-serpinA1 (serpinA1) or control vector pcDNA (vector) for overexpression of serpinA1 (A) or transfected with serpinA1 siRNA (siSerpinA1) or non-targeting siRNA (siNT) for knockdown (D). mRNA levels and secretion of MMP8 were quantified using real-time PCR ( $B$ and $\mathbf{E}$ ) and ELISA (C and F), respectively. ${ }^{\star} P<0.05$.
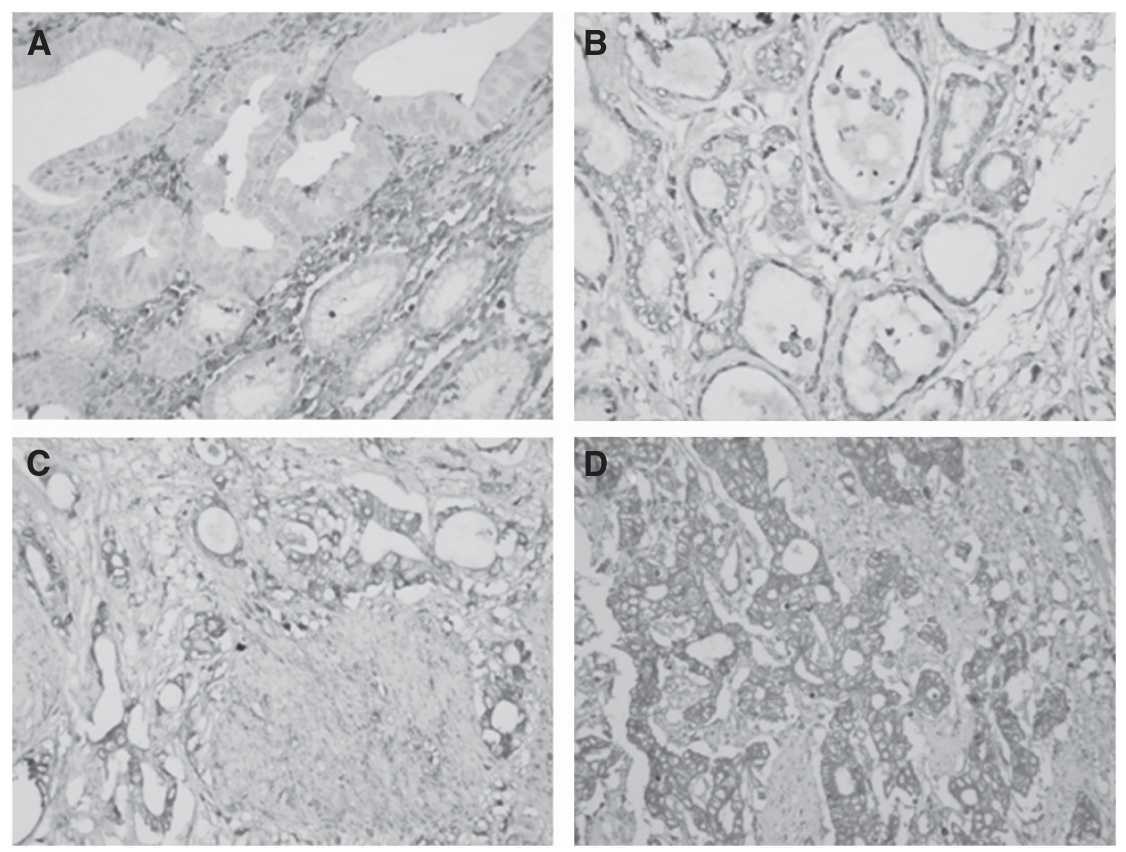

Figure 4. SerpinA1 expression in gastric cancer tissue samples. Intensity of serpinA1 immunostaining in tumour cells was scored as follows: negative $(-)(\mathbf{A})$, weak $(+)(\mathbf{B})$, moderate $(++)(\mathbf{C})$, and strong $(+++)(\mathbf{D})$. Stromal serpinA1 expression was scored as moderate $(++)(\mathbf{A})$, weak (C and D), or negative (B). Kaplan-Meier plots of overall survival according to serpinA1 expression. Magnification $\times 400$.

serpinA1 expression was increased in N1-stage cells $(22(40.0 \%))$, N2-stage cells (37 (59.7\%)), and N3-stage cells (36 (53.7\%)) relative to N0-stage cells (56 (25.9\%); $P<0.0001)$ (Table 1$)$. Similar to the patterns observed for serpinA1 expression in tumour cells, stromal serpin A1 expression was also associated with increased invasion depth ( $\mathrm{T}$ stage) $(P=0.001)$, perineural invasion $(P=0.036)$, lymphovascular tumour emboli $(P=0.011)$, and lymph node metastasis $(P=0.003)$ (Table 2$)$. These data indicate that serpinA1 overexpression in tumour cells and the stroma is correlated with clinical progression of gastric cancer, especially tumour invasion depth, perineural invasion, lymphovascular emboli, and lymph node metastasis.

In the survival analysis, the patient group survival rate was observed to decrease as tumour serpinA1 expression increased, in accordance with clinicopathologic data (Figure 5A). The average survival durations of the $(-),(+),(++)$, and $(+++)$ groups, which were classified according to serpinA1 expression levels, were $75.58,72.20,63.88$, and 61.64 months, respectively; this decrease was statistically significant $(P=0.001)$. These data suggest that serpinA1 expression is a prognostic predictor for patient survival of 


\begin{tabular}{|c|c|c|c|c|}
\hline \multicolumn{5}{|c|}{ Tumour serpinA1 expression } \\
\hline & No. & $(-)(+)$ & $(++)(+++)$ & $P$-value \\
\hline Age (years) & 400 & $58.2 \pm 0.72$ & $62.5 \pm 0.86$ & $<0.0001$ \\
\hline Size $(\mathrm{cm})$ & 400 & $4.35 \pm 0.19$ & $5.27 \pm 0.24$ & 0.003 \\
\hline \multicolumn{5}{|l|}{ Sex } \\
\hline Male & 258 & $148(57.4)$ & $110(42.6)$ & \\
\hline Female & 142 & $101(71.1)$ & $41(28.9)$ & \\
\hline \multicolumn{5}{|l|}{ Location } \\
\hline Upper & 43 & $23(53.5)$ & $20(46.5)$ & 0.113 \\
\hline Middle & 129 & $89(69.0)$ & 40 (31.0) & \\
\hline Lower & 228 & $137(60.1)$ & $91(39.9)$ & \\
\hline \multicolumn{5}{|l|}{ Gross type ${ }^{a}$} \\
\hline Elevated & 91 & $44(48.4)$ & $47(51.6)$ & $<0.0001$ \\
\hline Flat/depressed & 151 & $126(83.4)$ & 25 (16.6) & \\
\hline Excavated & 158 & $79(50.0)$ & $79(50.0)$ & \\
\hline \multicolumn{5}{|c|}{ Histological type ${ }^{b}$} \\
\hline Intestinal & 229 & $127(55.5)$ & $102(44.5)$ & 0.002 \\
\hline Diffuse & 167 & $119(71.3)$ & $48(28.7)$ & \\
\hline Mixed & 4 & $3(75.0)$ & $1(25.0)$ & \\
\hline \multicolumn{5}{|c|}{ Invasion depth ${ }^{c}$} \\
\hline T1 & 189 & $145(76.7)$ & $44(23.3)$ & $<0.0001$ \\
\hline T2 & 61 & $32(52.5)$ & $29(47.5)$ & \\
\hline T3 & 64 & $28(43.8)$ & $36(56.2)$ & \\
\hline T4 & 86 & $44(51.2)$ & $42(48.8)$ & \\
\hline \multicolumn{5}{|c|}{ Perineural invasion } \\
\hline Negative & 243 & $168(69.1)$ & 75 (30.9) & $<0.0001$ \\
\hline Positive & 157 & $81(51.6)$ & $76(48.4)$ & \\
\hline \multicolumn{5}{|c|}{ Lymphovascular emboli } \\
\hline Negative & 250 & $178(71.2)$ & $72(28.8)$ & $<0.0001$ \\
\hline Positive & 150 & $71(47.3)$ & $79(52.7)$ & \\
\hline \multicolumn{5}{|c|}{ Lymph node metastasis $^{d}$} \\
\hline NO & 216 & $160(74.1)$ & $56(25.9)$ & $<0.0001$ \\
\hline N1 & 55 & $33(60.0)$ & $22(40.0)$ & \\
\hline N2 & 62 & $25(40.3)$ & $37(59.7)$ & \\
\hline N3 & 67 & $31(46.3)$ & $36(53.7)$ & \\
\hline \multicolumn{5}{|c|}{ 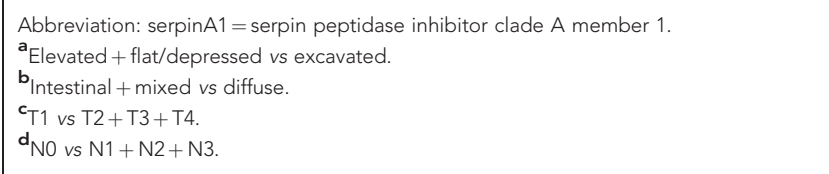 } \\
\hline
\end{tabular}

gastric cancer. However, stromal serpinA1 expression was not associated with poor outcomes, although it showed a tendency of decrease of survival in accordance with increased stromal serpinA1 expression (Figure 5B).

\section{DISCUSSION}

The onset and progression of gastric cancer are precipitated by various genetic alterations. Identifying proteins with tumourigenic properties and related proteins in gastric cancer cells is helpful in
Table 2. Relationship between stromal serpinA1 expression and clinicopathologic characteristics in 400 patients with gastric cancer

\begin{tabular}{|l|c|c|c|c|c|}
\cline { 3 - 5 } \multicolumn{5}{|c|}{ Stromal serpinA1 expression } \\
\hline & No. & $(-)$ & $(+)$ & $(++)$ & $P$-value \\
\hline Age (years) & 400 & $56.9 \pm 1.29$ & $60.2 \pm 0.64$ & $62.9 \pm 2.21$ & $<0.0001$ \\
\hline Size (cm) & 400 & $4.37 \pm 0.34$ & $4.76 \pm 0.18$ & $4.89 \pm 0.42$ & 0.003 \\
\hline Sex & & & & &
\end{tabular}

\begin{tabular}{l|l|l|l|l|l|}
\hline Sex \\
\hline Male & 258 & $57(22.1)$ & $179(69.4)$ & $22(8.5)$ & 0.274 \\
Female & 142 & $22(15.5)$ & $108(76.1)$ & $12(8.5$ & \\
\hline
\end{tabular}

Location $^{\text {a }}$

\begin{tabular}{|l|r|r|r|r|r|}
\hline Upper & 43 & $7(16.3)$ & $28(65.1)$ & $8(18.6)$ & 0.368 \\
Middle & 129 & $29(22.5)$ & $90(69.8)$ & $10(7.8)$ & \\
Lower & 228 & $43(18.9)$ & $169(74.1)$ & $16(7.0)$ & \\
\hline
\end{tabular}

\section{Gross type}

\begin{tabular}{|l|r|c|r|c|c|}
\hline Elevated & 91 & $7(7.7)$ & $73(80.2)$ & $11(12.1)$ & $<0.0001$ \\
Flat/depressed & 151 & $49(32.5)$ & $94(62.3)$ & $8(5.3)$ & \\
Excavated & 158 & $23(14.6)$ & $120(75.9)$ & $15(9.5)$ & \\
\hline
\end{tabular}

Histologic type ${ }^{b}$

\begin{tabular}{|l|r|r|r|r|r|}
\hline Intestinal & 229 & $35(15.3)$ & $172(75.1)$ & $22(9.6)$ & 0.065 \\
Diffuse & 167 & $42(25.1)$ & $113(67.7)$ & $12(7.2)$ & \\
Mixed & 4 & $2(50.0)$ & $2(50.0)$ & $0(0.0)$ & \\
\hline
\end{tabular}

\section{Invasion depth ${ }^{\mathrm{c}}$}

\begin{tabular}{|l|r|c|r|r|r|}
\hline T1 & 189 & $54(28.6)$ & $123(65.1)$ & $12(6.3)$ & $<0.0001$ \\
T2 & 61 & $5(8.2)$ & $53(86.9)$ & $3(4.9)$ & \\
T3 & 64 & $9(14.1)$ & $46(71.9)$ & $9(14.1)$ & \\
T4 & 86 & $11(12.8)$ & $65(75.6)$ & $10(11.6)$ & \\
\hline
\end{tabular}

\section{Perineural invasion}

\begin{tabular}{|l|l|l|l|l|l|}
\hline Negative & 243 & $58(23.9)$ & $166(68.3)$ & $19(7.8)$ & 0.036 \\
Positive & 157 & $21(13.4)$ & $121(77.1)$ & $15(9.6)$ & \\
\hline
\end{tabular}

\section{Lymphovascular emboli}

\begin{tabular}{|l|l|l|l|l|l|}
\hline Negative & 250 & $61(24.4)$ & $169(67.6)$ & $20(8.0)$ & 0.011 \\
Positive & 150 & $18(12.0)$ & $118(78.7)$ & $14(9.3)$ & \\
\hline
\end{tabular}

\section{Lymph node metastasis ${ }^{d}$}

\begin{tabular}{|l|r|c|r|r|r|}
\hline N0 & 216 & $55(25.5)$ & $148(68.5)$ & $13(6.0)$ & 0.003 \\
N1 & 55 & $11(20.0)$ & $38(69.1)$ & $6(10.9)$ & \\
N2 & 62 & $5(8.1)$ & $47(75.8)$ & $10(16.1)$ & \\
N3 & 67 & $8(11.9)$ & $54(80.6)$ & $5(7.5)$ & \\
\hline
\end{tabular}

Abbreviation: serpinA $1=$ serpin peptidase inhibitor clade $\mathrm{A}$ member 1.

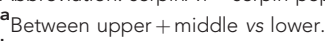

$\mathbf{b}_{\text {Intestinal }}+$ mixed vs diffuse.

cBetween $\mathrm{T} 1$ vs $\mathrm{T} 2+\mathrm{T} 3+\mathrm{T} 4$.

${ }^{d}$ Between No vs N1 + N2 + N3

understanding tumour progression, in discovering novel biomarkers, and in determining effective therapies for use in clinical settings. The present study showed that serpinA1 serves as a prognostic predictor for progression and patient survival of gastric cancer.

The expression of serpinA1, which codes for $\alpha$-1antitrypsin, a major plasma serpin that has a broad inhibitory spectrum against serine proteases, has been associated with the invasive potential of gastric, lung, and colorectal adenocarcinomas (Tahara et al, 1984; Karashima et al, 1990; Higashiyama et al, 1992). Snail has been found to have a role in the pathogenesis of several malignancies, 

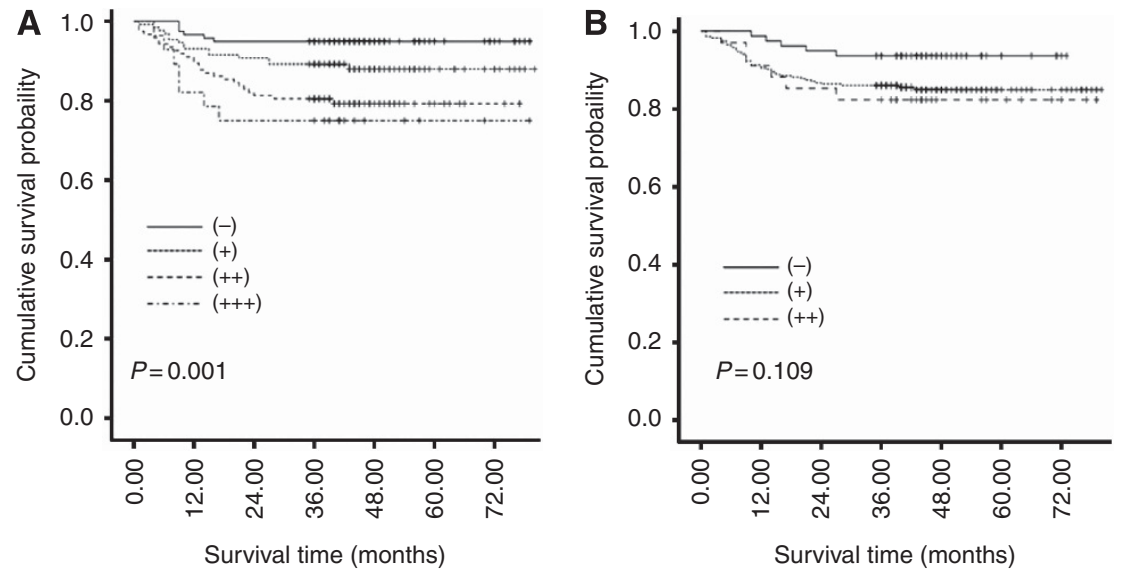

Figure 5. A linear relationship was observed between increased tumour serpinA1 expression and shortened survival overall in gastric cancer $(P=0.001)$. (A) Stromal serpinA1 expression was not significantly correlated with survival $(P=0.109)$. (B) The log-rank test was used to calculate the $P$-value.

predominantly in enhancing invasiveness and metastatic behaviour (Miyoshi et al, 2005; Olmeda et al, 2007). In our previous study, we reported that serpinA1 was upregulated in Snail-overexpressing gastric cancer patients (Shin et al, 2012). Consistent with previous results, here we observed that serpin $\mathrm{A} 1$ is markedly upregulated in the Snail-overexpressing gastric cancer cell lines MKN45 and AGS. Moreover, the results of the ChIP assay showed that Snail may increase serpinA1 protein expression by directly binding its promoter. Although the effect of serpinA1 has been studied in several cancers, the expression of serpinA1 has not been reported to be correlated with Snail. These results represent the first report, to the best of our knowledge, that serpinA1 is regulated by Snail. This may help to elucidate one of the many mechanisms by which serpinA1 is overexpressed in various cancers.

Despite its clinical relevance, much remains unknown regarding the functional role of serpinA1 in tumour cells. The function of serpinA1 in gastric cancer was identified by regulating its expression in the gastric cancer cell lines MKN45 and AGS. Results of transfection with the pcDNAserpinAlvector showed significantly increased migration and invasion of the serpinA1overexpressing cell lines relative to the control group. Conversely, when serpinAl expression was decreased using siRNA, cell migration and invasion decreased significantly. Results of the in vitro experiment herein showed that serpinA1 has an important role in migration and invasion of gastric cancer cells. These findings suggest that serpinA1 affects the incidence and progression of gastric cancer by promoting the mobility and metastasis of cancer cells. Our results are in concordance with other studies, which reported that serpinAl has a role in tumour progression (Tahara et al, 1984; Karashima et al, 1990; Higashiyama et al, 1992; Allgayer et al, 1998; de Sá et al, 2007; Normandin et al, 2010; Farshchian et al, 2011).

Although the mechanism of serpinA1 in tumorigenesis is not fully understood, previous studies have identified several mechanisms of tumorigenesis. It has been reported that tumour cells can produce and release not only a native form but also cleaved and/or degraded forms of serpinA1, which seem to be related to tumoural invasiveness (Kataoka et al, 1999). Kataoka et al (1999) reported that a $\mathrm{COOH}$-terminal fragment of serpinA1 increases tumour growth, possibly because of its inhibitory effects on the activity of natural killer cells against tumour cells (Congote and Temmel, 2004; Zelvyte et al, 2004). Another study showed that serpinA1 has an antiapoptotic effect on lung endothelial cells via inhibition of caspase-3 activity (Petrache et al, 2006). Metalloproteinases are key players in tumour growth and dissemination. The present results show that serpinA1 upregulates MMP8 expression in gastric cancer cells, but did not alter MMP7 or MMP12 expression (data not shown). Remodelling of the extracellular matrix by collagenolytic enzymes such as MMP1, MM8, and MMP13, as well as other proteases, is required for invasion (Noël et al, 2012). Thus, our results suggest that serpinA1 may contribute to gastric cancer progression by mediating MMP8.

In the present study, statistical analysis of immunohistochemical staining for serpinAl was used to determine its relationship with various critical clinicopathologic features in 400 gastric cancer patients. Serpin peptidase inhibitor clade A member 1 was frequently detected in tumour cells. In normal cells, negative or weak staining for serpinA1 was detected (Supplementary Figure). Serpin peptidase inhibitor clade A member 1 overexpression in tumour cells was correlated with high mean age and large tumour size. Serpin peptidase inhibitor clade A member 1 was overexpressed in excavated-type, mixed-type, and intestinal-type tumours. Moreover, serpinA1 was more frequently overexpressed in T2-, T3-, and T4-stage gastric cancers than in T1-stage cancers. These findings reaffirm the stage-specific nature of serpinA1 overexpression in gastric cancer. Further, the increased frequency of serpinA1 expression in gastric cancers with perineural invasion, lymphovascular emboli, and lymph node metastasis indicates that its expression affects gastric cancer onset and progression. These data are consistent with the significant role of serpinAl in invasion and migration of gastric cancer cell lines. Furthermore, comparison of serpinA1 expression and overall survival rate showed that survival rate was lower in patients with serpinA1 overexpression, indicating serpinAl is a prognostic predictive factor of gastric cancer. Similarly, enhanced serpinA1 expression has been reported to be correlated with poor prognoses in colorectal cancer, lung cancer, squamous cell cancer, and human leukocyte antigenpositive cervical cancer patients (Karashima et al, 1990; Higashiyama et al, 1992; Allgayer et al, 1998; Kloth et al, 2008). Moreover, many studies have indicated that serpinAl expression is correlated with the clinicopathologic features of tumours, including the depth of tumour invasion and the presence of lymph node and distant metastases. Tahara et al (1984) also reported increased serpinA1 expression in well-differentiated-type gastric cancers and an association of this expression with prognosis, although they only discussed the relationship between serpinAl expression and the histologic type of cancer, and the effect of serpinA1 expression on 2-year survival.

Serpin peptidase inhibitor clade A member 1 expression also was increased in the stroma adjacent to tumours. Stromal staining 
for serpinA1 has been reported to represent mainly serum-derived serpinA1 produced in the liver (Farshchian et al, 2011). In the present study, we found statistically significant correlation between serpinA1 expression in tumour cells and in the stroma by Spearman's rank test. Therefore, serpinA1 may also be secreted from tumour cells to surrounding tissue (Chang et al, 2012). Serpin peptidase inhibitor clade A member 1 expression in the stroma was related to gastric cancers with perineural invasion, lymphovascular emboli, and lymph node metastasis, indicating that stromal serpinA1 is also associated with gastric cancer progression. These results are consistent with those from tumour cells. However, no relationship between stromal serpin $\mathrm{A} 1$ and prognosis was observed, which differs from our results for serpinA1 expression in tumour cells. These results suggest that the presence of serpinA1 in gastric tumour cells or the stroma is related to the invasive growth of the tumours.

Collectively, our results contribute to an improved understanding of the mechanism by which serpinA1 is involved in the progression of gastric cancer cells. These results suggest that serpinA1 expression could provide information regarding clinical stage and lymph node metastasis, and that it may therefore be a potentially useful prognostic biomarker for patients with gastric cancer.

\section{ACKNOWLEDGEMENTS}

This work was supported by a grant (0920050) from the National R\&D Program for Cancer Control, Ministry for Health, Welfare and Family Affairs, Republic of Korea. The biospecimens for this study were provided by the Pusan National University Hospital or by members of the National Biobank of Korea, which is supported by the Ministry of Health, Welfare, and Family Affairs.

\section{CONFLICT OF INTEREST}

The authors declare no conflict of interest.

\section{REFERENCES}

Allgayer H, Babic R, Grützner KU, Beyer BC, Tarabichi A, Schildberg FW, Heiss MM (1998) Tumor-associated proteases and inhibitors in gastric cancer: analysis of prognostic impact and individual risk protease patterns. Clin Exp Metast 16: 62-73.

Brantly M (2002) Alphal-antitrypsin: not just an antiprotease: extending the half-life of a natural anti-inflammatory molecule by conjugation with polyethylene glycol. Am J Respir Cell Mol Biol 27: 652-654.

Chang YH, Lee SH, Liao IC, Huang SH, Cheng HC, Liao PC (2012) Secretomic analysis identifies alpha- 1 antitrypsin (A1AT) as a required protein in cancer cell migration, invasion, ans pericellular fibronictin assembly for facilitating lung colonization of lung adenocarcinoma cells. Mol Cell Proteom 11: 1320-1339.

Congote LF, Temmel N (2004) The C-terminal 26-residue peptide of serpin A1 stimulates proliferation of breast and liver cancer cells: role of protein kinase C and CD47. FEBS Lett 576: 343-347.

de Sá SV, Corrêa-Giannella ML, Machado MC, Krogh K, de Almeida MQ, Albergaria Pereira MA, Coelho Siqueira SA, Patzina RA, Ibuki FS, Sogayar MC, Machado MC, Giannella-Neto D (2007) Serpin peptidase inhibitor clade A member 1 as a potential marker for malignancy in insulinomas. Clin Cancer Res 13: 5322-5330.

Edge SB, Byrd DR, Compton CC, Fritz AG, Greene FL, Trotti A (2009) American Joint Committee on Cancer Staging Manual. 7th edn. Springer: New York, Y, USA.

Farshchian M, Kivisaari A, Ala-Aho R, Riihilä P, Kallajoki M (2011) Serpin peptidase inhibitor clade A member 1 (SerpinA1) is a novel biomarker for progression of cutaneous squamous cell carcinoma. Am J Pathol 179: $1110-1119$.
Higashiyama M, Doi O, Kodama K, Yokouchi H, Tateishi R (1992) An evaluation of the prognostic significance of alpha-1-antitrypsin expression in adenocarcinomas of the lung: an immunohistochemical analysis. Br J Cancer 65: 300-302.

Janciauskiene S (2001) Conformational properties of serine proteinase inhibitors (serpins) confer multiple pathophysiological roles. Biochim Biophys Acta 1535: 221-235.

Karashima S, Kataoka H, Itoh H, Maruyama R, Koono M (1990) Prognostic significance of alpha-1-antitrypsin in early stage of colorectal carcinomas. Int J Cancer 45: 244-250.

Kataoka H, Uchino H, Iwamura T, Seiki M, Nabeshima K, Koono M (1999) Enhance tumor growth and invasiveness in vivo by a carboxyl-terminal fragment of a1-proteinase inhibitor generated by matrix metallo proteinases: a possible modulatory role in natural killer cytotoxicity. Am J Pathol 154: 457-468.

Kim DH, Shin N, Kim GH, Song GA, Jeon TY, Lauwers GY, Park do Y (2013) Mucin expression in gastric cancer: repparaisal of its clinicopathological and prognostic significance. Arch Pathol Lab Med 137: 1047-1053.

Kim WH, Park CK, Kim YB, Kim YW, Kim HG, Bae HI, Song KS, Chang HK, Chang HJ, Chae YS (2005) A standardized pathology report for gastric cancer. Korean J Pathol 39: 106-113.

Kitano E, Kitamura H (1993) Synthesis of the third component of complement (C3) by human gastric cancer-derived cell lines. Clin Exp Immunol 94: 273-278.

Kloth JN, Gorter A, Fleuren GJ, Oosting J, Uljee S, ter Haar N, Dreef EJ, Kenter GG, Jordanova ES (2008) Elevated expression of serpinA1 and SerpinA3 in HLA-positive cervical carcinoma. J Pathol 215: 222-230.

Kovoor PA, Hwang P (2009) Treatment of resectable gastric cancer: current standards of care. Expert Rev Anticancer Ther 9: 135-142.

Law RH, Zhang Q, MxGowan S, Buckle AM, Silverman GA, Wong W, Rosado CJ, Langendorf CG, Pike RN, Bird PI, Whisstock JC (2006) An overview of the serpin superfamily. Genome Biol 7: 216.

Miyoshi A, Kitajima Y, Kido S, Shimonishi T, Matsuyama S, Kitahara K, Miyazaki K (2005) Snail accelerates cancer invasion by upregulating MMP expression and is associated with poor prognosis of hepatocellular carcinoma. Br J Cancer 92: 252-258.

Noël A, Gutiérrez-Fernández A, Sounni NE, Behrendt N, Maquoi E, Lund IK, Cal S, Hoyer-Hansen G, López-Otín C (2012) New and paradoxical roles of matrix metalloproteinases in the tumor microenvironment. Front Pharmacol 3: 140.

Normandin K, Péant B, Le Page C, de Ladurantaye M, Ouellet V, Tonin PN, Provencher DM, Mes-Masson AM (2010) Protease inhibitor SERPINA1 expression in epithelial ovarian cancer. Clin Exp Metastasis 27: $55-69$.

Olmeda D, Moreno-Bueno G, Flores JM, Fabra A, Portillo F, Cano A (2007) SNAI1 is required for tumor growth and lymph node metastasis of human breast carcinoma MDA-MB-231 cells. Cancer Res 67: 11721-11731.

Parkin DM, Bry F, Ferlay J, Pisani P (2005) Global cancer statistics, 2002. CA Cancer J Clin 55: 74-108.

Petrache I, Fijalkowska I, Medler TR, Skirball J, Cruz P, Zhen L, Petrache HI, Flotte TR, Tuder RM, Cruz P (2006) Alpha-1 antitrypsin inhibits caspase- 3 activity, preventing lung endothelial cell apoptosis. Am J Pathol 169: 1155-1166.

Roukos DH, Kappas AM (2005) Perspectives in the treatment of gastric cancer. Nat Clin Pract Oncol 2: 98-107.

Shin NR, Jeong EH, Choi CI, Moon HJ, Kwon CH, Chu IS, Kim GH, Jeon TY, Kim DH, Lee JH, Park do Y (2012) Overexpression of Snail is associated with lymph node metastasis and poor prognosis in patients with gastric cancer. BMC Cancer 12: 521.

Siverman GA, Bird PI, Carrell RW, Church FC, Coughlin PB, Gettins PG, Irving JA, Lomas DA, Luke CJ, Moyer RW, Pemberton PA, RemoldO'Donnell E, Salvesen GS, Travis J, Whisstock JC (2001) The serpins are an expanding superfamily of structurally similar but functionally diverse proteins. Evolution, mechanism of inhibition, novel functions, and a revised nomenclature. J Biol Chem 276: 33293-33296.

Tahara E, Ito H, Taniyama K, Yokozaki H, Hata J (1984) Alpha 1-antitrypsin, alpha 1-antichymotrypsin, and alpha 2-macroglobulin in human gastric carcinomas: a retrospective immunohistochemical study. Hum Pathol 15: 957-964. 
Thiery JP, Acloque H, Huang RYJ, Mieto MA (2009) Epithelicalmesenchymal transitions in development and disease. Cell 139: 871-890.

Zelvyte I, Sjögren HO, Janciauskiene S (2002) Effects of native and cleaved forms of alpha1-antitrypsin on ME 1477 tumor cell functional activity. Cancer Detect Prev 26: 256-265.

Zelvyte I, Stevens T, Westin U, Janciauskiene S (2004) alpha1-antitrypsin and its C-terminal fragment attenuate effects of degranulated neutrophil-conditioned medium on lung cancer HCC cells, in vitro. Cancer Cell Int 4: 7.

This work is published under the standard license to publish agreement. After 12 months the work will become freely available and the license terms will switch to a Creative Commons AttributionNonCommercial-Share Alike 3.0 Unported License.

Supplementary Information accompanies this paper on British Journal of Cancer website (http://www.nature.com/bjc) 